\title{
Politique et poétique de l'interface dans l'histoire littéraire africaine
}

Jean Eudes BIEM

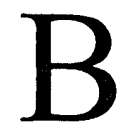
ientôt un siècle de production et de critique des littératures africaines en langues européennes et le discours d'une histoire littéraire africaine ne va toujours pas de soi. Comment opérer des délimitations séquentielles dans cette littérature sans tomber dans les travers de l'historicisation discriminatoire, sans historiciser seulement l'écriture postcoloniale, avec tout ce que cela comporte comme corollaires? Comment historiciser aussi les littératures africaines des périodes précoloniales généralement fossilisées ici et là dans l'uniformisme d'une équivoque "oralité première", terme réductionniste sans rupture conceptuelle suffisante avec la conception impériale d'une Afrique dénuée d'écriture et d'historicité ? Malgré les efforts de nombre de chercheurs, il est certain que ce problème ne sera résolu de façon satisfaisante que dans des travaux futurs dont l'exécution sera rendue encore plus difficile par la distance, l'épuisement des pratiques de l'oralité et la raréfaction croissante des sources de première main. Ne traitant de ces questions que de manière connexe, la présente étude n'a aucune prétention à résoudre ce problème. En revanche, elle met en évidence l'esquisse d'une trajectoire de progression dans les usages africains des ressources culturelles pour la construction d'un discours de reprise de l'initiative historique au vingtième siècle. La lecture des rythmes parallèles des faits sociopolitiques et littéraires ici proposée vise à dégager non seulement 
les tendances organiques de l'écriture, mais aussi la dynamique sous jacente de développement autonome que la littérature réussit à sa manière et présente comme traductible dans la sphère politique. La réappropriation de l'initiative exigeant une organisation discriminatoire à la fois des emprunts et des ressources endogènes, l'imaginaire se situe à l'interface de ces deux sources. C'est cette poétique et cette politique de l'interface, ce travail dans les interstices de l'emprunté et de l'endogène, tantôt spontané, tantôt stratégiquement orienté, qui sera conceptualisé ici en termes d'hybridité.

Une telle tentative exige de poser comme centraux deux faits : que cette littérature est un moyen d'accès privilégié aux réalités africaines, et qu'en écrivant dans les langues occidentales, les Africains sont influencés par leurs langues maternelles et se retrouvent ainsi en situation de diglossie, voire de multilinguisme. De nombreux effets esthétiques découlent de cette " écriture polyglotte ", ' et les études consacrées à ces effets esthétiques montrent que cette écriture tire sa valeur de la double appartenance qui a présidé à sa naissance, ainsi que le confirme le linguiste Mwatha Ngalasso (1984):

La littérature africaine, de langue française, située à l'intersection de plusieurs langues, de plusieurs traditions littéraires et de plusieurs cultures, porte nécessairement la marque d'une double appartenance. Elle est incontestablement africaine en tant que lieu d'expression authentique d'une sensibilité, d'une affectivité et d'un intellect africains elle est française en tant que parole élaborée en langue française dans une écriture à la fois plurielle et multirelationnelle (34).

Voilà qui situe irréductiblement cette écriture dans l'hybridité, repérée ici à partir d'une relecture synthétique des travaux d'histoire littéraire africaine. ${ }^{2}$ L'analyse des enjeux et modalités de cette localisation suit un schéma globalement diachronique et met un accent particulier sur le roman, genre dominant qui restitue avec acuité le projet de développement inscrit au cœur de la litterature africaine. La narration de l'espace public mobilise tous les enjeux mis en évidence par Edward Saïd (1994):

The appropriation of history, the historicization of the past, the narrativization of the society, all of which give the novel its force, include the accumulation and differentiation of social space, space to be used for social purposes (78).

Le roman transpose l'histoire, s'y intègre et y contribue, notamment en ajoutant de la densité à la texture identitaire. Relire l'histoire littéraire africaine sous l'angle de l'hybridité est d'autant plus pertinent que cette 
littérature est, au vingtième siècle, le fait des couches les plus hybridées, l'expression des élites occidentalisées du continent.

\section{Cohabitation et dichotomie des littératures de soumission et de résistance}

Les spécialistes s'accordant sur le fait que la littérature africaine caractéristique de l'époque dite néocoloniale ne commence véritablement qu'à la veille des années 1970, la période coloniale englobe les œuvres parues jusqu'en $1967 .{ }^{3}$ Ici, les deux attitudes fondamentales reflètent les positions des colonisés Ariel et Caliban face au colonisateur Prospéro dans Une Tempête d'Aimé Césaire : tandis que Ariel opte pour la collaboration, Caliban décide de combattre. Autant certaines œuvres sont anticolonialistes, autant d'autres revêtent des attitudes respectueuses, voire promotrices de l'ordre colonial. Mais il arrive aussi que soumission et résistance cohabitent chez le même écrivain ou dans le même ouvrage. Du reste, même naïve, l'imitation est en soi une attitude de soumission au même titre que l'acceptation consciente de la domination, en tant qu'elle emporte souscription aux visées de la culture dominante. Le colonisateur attendant du colonisé qu'il adopte les formes et intériorise les valeurs et normes de la puissance dominatrice, l'imitation satisfait aux exigences du projet central de la "mission civilisatrice", consistant à transformer la culture du colonisé par la reprise ou la copie de la culture du colonisateur.

Cette relation dichotomique - soumission et résistance à l'intérieur et entre les ouvrages - apparaît dans la lecture consensuelle des deux premiers romans négro-africains les plus connus, lecture que j'emprunte à Pius Ngandu Nkashama (1979):

Le roman de René Maran, Batouala, significativement soustitré « véritable roman nègre », tentera désespérément de dissiper les ombres fuligineuses des mythes coloniaux. Sans doute, Maran cède-t-il, lui aussi (volontairement ou non), à ces mêmes mythes qui relèvent davantage de l'imagerie populaire et d'une mentalité rousseauiste européenne dans la description de certaines scènes : danses, rites d'initiation [. . .], rigueur dans les mœurs et les traditions, promiscuité, brutalité sauvage dans la manifestation de certains instincts, etc. Néanmoins, son œuvre constitue un premier acte de courage posé par un écrivain noir en face de l'hydre de la colonisation [. . . ]. C'est aussi dans ce sens qu'il faut comprendre le roman de Bakary Diallo, Force Bonté, publié en 1925. Pour cet intellectuel sénégalais, il s'agit de réfuter les arguments de la littérature coloniale, arguments 
selon lesquels le colonisateur européen, en débarquant en Afrique, se serait trouvé en face de sociétés inorganisées, sans aucun passé historique. Mais, curieusement, les affirmations de Bakary Diallo semblent bien timides, et même se circonscrivent parfaitement à l'intérieur du cercle idéologique colonialiste. Diallo en arrive à exalter l'œuvre civilisatrice en elle-même, par sa dimension salvatrice... (49).

La dichotomie signalée se double ici d'un contraste dans la qualité de l'écriture. Tout se passe comme si l'esthétique établit une justice historique, car Batouala (1921) se distingue par un anticolonialisme violent et une excellente facture littéraire, tandis que Force Bonté (1925) fait "l'éloge du colonisateur dans un style décousu, primaire et d'une consternante puérilité ", ainsi que le souligne par ailleurs Boubacar Boris Diop $(1999,6)$. Mais une quête aussi monumentale ne va pas sans ambiguïtés, erreurs d'appréciation et quiproquos. Du point de vue interne, Maran se retrouve lui aussi piégé par les clichés de l'africanisme eurocentriste, tandis que Diallo prétend réfuter précisément ces clichés. On retrouvera plus tard cette ambiguïté en poésie, chez Aimé Césaire et Léopold Sédar Senghor.

À partir des années 1930, comme pour réagir à ce tiraillement, certains romanciers, à l'instar du Sénégalais Ousmane Socé Diop, s'engagent dans un traitement très explicite de l'hybridité qui en est le fondement. Jacques Chevrier (1984) observe que les romans De Socé Diop, Karim (1935) et Mirages de Paris (1937), exaltent d'un même mouvement le métissage et la subalternéité :

Ainsi, tout en demeurant profondément Sénégalais par son attachement à un mode de vie spécifiquement africain - fêtes, manières d'être ensemble, coutumes et traditions musulmanes - Ousmane Socé admet inconditionnellement la suprématie de la civilisation européenne et, loin de dénoncer les abus pourtant éclatants, recommande à ses concitoyens de "prendre en exemple ces pays de vieille civilisation $»(29)$.

Comme on pouvait s'y attendre cette attitude attira à Ousmane Socé Diop les félicitations de Robert Delavignette, préfacier de Karim et Gouverneur de la France au Sénégal.

A l'opposé, bien que traitant plus explicitement encore de l'irréversible hybridité résultant de la rencontre coloniale, la plupart des romans ultérieurs, dont la désormais canonique Aventure ambiguë (1961) de Cheikh Hamidou Kane, évitent voire éventent avec succès le piège du larbinisme. Ils insistent davantage sur l'avenir commun résultant de 
l'hybridité et des influences. Le personnage du chef des Diallobé le signifie sans ambages à son interlocuteur français, Paul Lacroix: « nous n'avons pas eu le même passé, vous et nous, mais nous aurons le même avenir, rigoureusement. » (92). Cheikh Hamidou Kane posait ainsi un principe fondamental de la théorisation de l'hybridité dans le domaine du postcolonialisme: "The history of the West and the history of the nonWest are by now irrevocably different and irrevocably shared. Both have shaped and been shaped by each other in specific and specifiable ways». ${ }^{4}$

Nullement fortuite, la lucidité de Hamidou Kane résulte d'une accumulation d'effets sertis dans le métal de la littérature anticolonialiste et militante des années 1950 qui accompagnait les processus dits de décolonisation. Sur le modèle balzacien, le roman de cette époque s'attelle à restituer l'histoire coloniale et à en dénoncer les servitudes, présentant des séries de tableaux révoltants de la seule réalité africaine objective, la condition des colonisés dominés, humiliés, résignés. Les romans les plus représentatifs de cette tendance sont les trilogies des Camerounais Mongo Béti : Ville cruelle (1954), Le Pauvre Christ de Bomba (1956), Le Roi miraculé (1958) et Ferdinand Oyono : Une Vie de boy (1956), Le Vieux nègre et la médaille (1956), Chemin d'Europe (1960).

La volonté d'affirmation sous jacente à ce militantisme assure le lien de continuité entre les tendances littéraires des années 1950 et 1960. Pius Ngandu Nkashama résume ainsi cette période «autour des indépendances» :

En cette période, sont soulignées l'antithèse et l'antinomie entre le monde occidental et le monde africain, entre la culture blanche et l'univers spirituel des Noirs, entre la technique déshumanisante et la " paix dans les villages », l'accord primordial entre l'homme et la nature. Il en résulte des tensions entre les deux communautés [...]A l'intérieur du conflit et des tensions entre le colonisé et le colonisateur, entre l'administrateur blanc et l'ouvrier noir sousexploité, va s'en inscrire un autre, plus profond encore, entre la tradition et le modernisme, entre les jeunes et les vieux (50).

Ces tensions structurent également les œuvres poétiques, dont Epitomé (1962) et Le Ventre (1964) de Tchicaya U'Tamsi qui auront un retentissement particulier. Elles illustrent les relations entre histoire et textualité que Roland Barthes (1953) et Edward Said (1980) conceptualisent en termes de «solidarité», et réaffirment ainsi la fonctionnalité de nombre de traditions littéraires africaines. C'est le rappel que fera encore Barthélémy Kotchy au colloque des écrivains à Lagos en 1988 : «l'art pour l'art n'est pas de mise. L'art doit se mettre au service d'une cause, en 
l'occurrence le développement national. Et là, force est de constater que l'on retourne aux sources». ${ }^{5}$ Dans ce contexte dominé par une littérature de résistance et de contestation, il s'est instauré une critique militante que Aminata Sow Fall et Jean Blachère (1977) n'hésitent pas à qualifier de «dogmatique». D'après cette école dont Mongo Béti sera le plus célèbre défenseur parmi les écrivains, tout ouvrage qui manque d'engagement anticolonialiste s'apparente mutatis mutandis à la littérature exotique européenne et appartient à la littérature de soumission. Pour Alexandre Biyidi alias Mongo Béti, L 'Enfant noir (1953) de Camara Laye, qui prétend être un témoignage des réalités africaines de son époque sans mentionner le moindre abus de l'administration coloniale, est l'épitomé même de ce larbinisme complice et mensonger fait écriture. ${ }^{6}$

Développée dans un contexte de flottement identitaire, la volonté d'affirmation des Africains s'effectue dans un interface de temporalités caractérisé par leur tiraillement entre la nécessité de construire une Afrique nouvelle et la contemplation des anciennes valeurs refuges parfois fossilisées. De façon prévisible, une telle problématique identitaire n'aurait $\mathrm{pu}$, en aucune manière, éviter de poser le problème de l'authenticité. Or en situation d'hybridité postcoloniale, la recherche de l'authenticité est une attitude de repli identitaire. Cette attitude a présidé à la naissance du mouvement de la négritude que Edward Said (1994) identifie à juste titre comme un impressionnant phénomène nativiste (228). Ce paradoxe du nativisme hybride explique pourquoi les deux principaux promoteurs de la négritude s'opposent au sujet de l'hybridité. "Césaire rejette toute idée de métissage et revendique une âme (identité) nègre pure ("Esprit de brousse"), inaltérable par les contingences historiques [.. .]. Aux antipodes de Césaire, Senghor pose l'idée selon laquelle seul le métissage répond à la vérité historique. Le nègre en est le produit» (Ngal 1994, 21-22).

Au-delà des attitudes différencielles, la négritude vise indubitablement une appropriation nostalgique des valeurs nègres détruites par la colonisation, ainsi qu'en témoignent ces vers de Léon Gontran-Damas cités par Stanislas Adotévi dans Négritude et négrologues (1972) : «Me sentir moi même de ce que hier/J'étais hier/ Sans complexité / Hier » (33). Malgré son apport irremplaçable à la littérature, cette quête était pourtant vouée à l'échec, vu que la culture postcoloniale est inévitablement et irréversiblement hybride, autre truisme des théories postcoloniales :

Precolonial cultural purity can never be fully recovered. Postcolonial cultures are inevitably hybridised, involving a dialectical relationship between European ontology and epistemology and 
the impulse to create or recreate independent local identity. [...] It is not possible to create or recreate national or regional formations wholly independent of their historical implication in the European colonial enterprise (Ashcroft et al., : 1995, 95).

Il est donc impossible à Aimé Césaire de « récupérer l'antique silex déposé en lui par l'Afrique mais recouvert par les couches négrophobes successives de l'assimilation » (Ngal 23).

Rejetée ou non, l'assimilation actualise le rôle d'instance médiatrice joué le plus souvent par le discours de l'africanisme eurocentriste entre les Africains et le savoir sur l'Afrique et le monde. C'est bien à travers ces clichés que les poètes de la négritude connaissent l'Afrique dite authentique, ce qui conduit à l'incurable souffrance exprimée dans ces célèbres vers élégiaques de Léon Laleau : «Sentez- vous cette souffrance ? / Et ce désespoir à nul autre égal / D'apprivoiser avec les mots de France $/$ Ce cœur qui m'est venu du Sénégal ? ${ }^{7}$ En recourant à cette instance médiatrice, la négritude tend à prolonger paradoxalement l'idéologie coloniale. On en trouve plusieurs traces dans le Cahier d'un retour au pays natal qui est probablement le poème le plus révolutionnaire de tout le mouvement. Lorsque Césaire proclame : «nous vous haïssons vous et votre raison, nous nous réclamons de la démence précoce de la folie flamboyante du cannibalisme tenace » (73), il brave évidemment les thèses racistes de Kant, Nietzsche, Lévi-Brühl, Gobineau et bien d'autres, selon lesquelles le nègre se définit par la sauvagerie délirante et la mentalité prélogique. À ce niveau Césaire demeure encore irréprochable. Mais lorsqu'il décrit les nègres comme « Ceux qui n'ont inventé ni la poudre ni la boussole / Ceux qui n'ont jamais su dompter la vapeur ni l'électricité / Ceux qui n'ont exploré ni les mers ni le ciel » (111), comme pour citer le « sinistre Jules Romains » proclamant que l'Afrique « n'a encore donné et ne donnera jamais un Einstein, un Stravinsky, un Gerschwin » (Adotévi 36), son ironie et sa satire se teignent d'un auto rejet inconscient. Cheikh Anta Diop (1954) démontre à cet égard que la plus élémentaire déconstruction du Cahier d'un retour au pays natal prouve que, dans ses contradictions fondatrices, l'ouvrage est dominé lui aussi par les clichés africanistes fondés sur l'ignorance de l'histoire des civilisations africaines. S'il en est ainsi de Césaire, il devient indéniable que l'image du nègre vulgarisée par la négritude se ramène à celle créée par l'Occident impérialiste. Profondément révolutionnaire, Césaire demeure indéniablement prisonnier du mythe nègre, actualisant la conception gramscienne de l'hégémonie où, à travers l'éducation notamment, opèrent les dynamiques de domination par consentement. ${ }^{8}$ 
Face au caractère fallacieux de l'essentialisme de la négritude, Molefi Kete Asante (1990) revient sur la nécessité de reposer un sérieux problème méthodologique que le mouvement à négligé:

All methods of doing research have philosophical roots with specific assumptions about phenomena, human inquiry, and knowledge. The africanist's frame of reference has too often been eurocentric, that is, flowing from a conceptualization of African people devoloped to support the western version of Africa (23).

La revendication de l'africanité ne garantit nullement a priori une bonne connaissance des réalités africaines. D'ailleurs, la conscience de race qui fonde la négritude peut aussi être perçue comme un résultat du déracinement, une manifestation de l'hybridité. L'écrivain sud-africain Peter Abrahams postule que le racisme occidental est responsable, dans la psychologie africaine, de cette oscillation entre la honte et la fierté d'être noir:

Être noir a peu d'importance dans l'Afrique des tribus car le comportement racial y est sain et naturel. La couleur de la peau ne compte pas. C'est un don de Dieu qui n'est ni un privilège ni un handicap. [. . .] Ce n'était rien de plus avant que des conceptions étrangères ne viennent donner à la couleur de la peau un sens, une importance particulière. ${ }^{9}$

La négritude est donc un produit de l'histoire coloniale dans laquelle s'inscrivent la cohabitation raciale et la subalternéité qui accompagne l'hybridité chez les nègres.

La preuve en est que la négritude prolonge la Négro-renaissance de Harlem, apparue aux État-Unis dans les années 1920, un environnement de ségrégation raciale brutale et souvent meurtrière, où la conscience de race est des plus aiguës. Lylian Kesteloot (1981) signale en effet que le « véritable père de la négritude » c'est W. E. B. Dubois auteur du leitmotiv de la fierté noire qui deviendra la base de la négritude : « je suis nègre, écrit en 1890, William Dubois, et je me glorifie de ce nom; je suis fier du sang noir qui coule dans mes veines » (47). Du reste, comme la Négro-renaissance, la négritude visait bien à libérer les noirs de leur subalternéité, afin que la vieille négritude faite de soumission et de silence se « cadavérise » devant la « négritude qui se met debout ». Lancé dans le Cahier d'un retour au pays natal, ce projet césairien se poursuit dans La Tragédie du roi Christophe: « De noms de gloire je veux couvrir vos noms d'esclaves, de noms d'orgueil nos noms d'infamie, de noms de rachat nos noms d'orphelins ! C'est d'une nouvelle naissance, messieurs, 
qu'il s'agit! " (37). Au vu de ce projet, il faut convenir avec Stanislas Adotévi que « la négritude est politique avant d'être poétique » (57).

Par suite, il suffit d'observer les influences s'exerçant sur le travail de recherche esthétique de la négritude pour conclure de son hybridité. Certes les poètes, pour donner une impression d'authenticité, usent et abusent de symboles africains comme la rythmique du tam-tam, la symbolique des masques, la mystique des totems. Mais, les spécialistes de l'histoire littéraire africaine à l'instar de Georges Ngal, Pius Ngandu Nkashama, Lylian Kesteloot, etc., s'accordent avec Jacques Chevrier (1984) que Césaire, Senghor et les autres chantres de la négritude restent profondément tributaires du classicisme, du surréalisme et d'autres influences non nègres (53-88). Finalement la négritude n'aura été qu'une réaction au malaise qui accompagne l'hybridité chez les négro-africains dominés. Il importe de noter que le souci de libération politique et culturelle qui la fonde est resté une constante dans la littérature africaine, même après les indépendances.

Les limites n'étant pas étanches, certaines caractéristiques de la littérature africaine de l'époque coloniale persistent dans la période dite néo-coloniale. On note avec Olympe Bhêly-Quenum la continuité de « la transcription des réalités africaines $»^{10}$ qui instruit la convergence entre poétique et politique, entre littérature et transformation sociale. En outre, la prépondérance du roman s'affirme davantage. Cependant, des changements capitaux se manifestent. Ils se traduisent par une plus grande richesse thématique et formelle qui, avec d'autres innovations, se fonde sur le "travail" de l'hybridité, un ensemble développements épistémologiques, esthétiques et sociopolitiques élaborés sur les lignes de force d'une interstitialité plus stratégique, opérant sur la base des réalités structurées par des héritages de l'africanité et de l'européanité dont la reconfiguration transcende de loin les binarités.

\section{De l'hybridité dans les nouveautés thématiques}

La nouveauté littéraire dans l'Afrique indépendante part de 1968, avec Les Soleils des indépendances d'Amadou Kourouma et Le Devoir de violence de Yambo Ouologuem, deux romans qui inscrivent de nouveaux rapports entre les écrivains et le contexte global de l'espace africain. Le nouveau contexte se caractérise par la conscience de l'échec des indépendances qui entraîne le procès du néocolonialisme et de ses "fondés de pouvoir" responsables des sociétés de misère, de corruption et d'oppression au-dessus lesquelles trônent les bourgeoisies nationales. 
La critique de ces sociétés est au coeur de la littérature dite du désenchantement:

Avec Le devoir de violence, de Yambo Ouologuem, Les soleils des indépendances d'Amadou Kourouma ou Remember Ruben, de Mongo Béti, les auteurs francophones ont entrepris de dresser, à leur tour, et à des degrés divers, un réquisitoire sévère et une satire acerbe à l'encontre des mœurs politiques de l'Afrique contemporaine. Corruption, népotisme, vénalité, despotisme ou incurie des dirigeants et de leurs complices y sont dénoncés sans ambages (Chevrier 1984, 135).

Parmi les romans qui se distinguent par cette critique sociopolitique il faut signaler en priorité Le Cercle des tropiques (1972) et le Récit du cirque... de la vallée des morts (1975) d'Alioum Fantouré, Perpétue et l'habitude du malheur et Remember Ruben (1974) de Mongo Béti, Entre les eaux (1973) de ValentinYves Mudimbe, ainsi que La Carte d'identité de Jean-Marie Adiaffi, La Vie et demie de Sony Labou Tansi, Les Crapauds-brousse de Thierno Monénembo, Le Jeune homme de sable de Williams. Sassine et L'Errance de Mbwil a Mpang Ngal, tous publiés en 1979, etc. Cette thématique domine jusqu'au début des années 1980 , avec Le Temps de Tamango (1981) de Boubacar Boris Diop, Le Pleurerrire (1982) de Henri Lopès, $L$ 'État honteux (1981) et $L$ 'Anté peuple (1983) de S.L. Tansi, ainsi que Elle sera de jaspe et de corail (1983) de Werewere Liking.

Tous ces romans et bien d'autres critiquent les élites occidentalisées qui, souffrant d'extériorité du fait de leur hybridité mal assumée, dirigent les pays africains à la manière des administrateurs coloniaux qui furent leurs maîtres avant les indépendances, vérifiant un proverbe bien connu en Afrique : « on n'est jamais que l'enfant de son école ». La bourgeoisie nationale qui gère l'échec du placage de l'État d'origine européenne et ses conséquences néfastes, pérennise donc un certain ordre colonial, exacerbant ainsi un potentiel tyrannique dans lequel Bart Moore-Gilbert (1998) voit un véritable avertissement politique en rapport avec la problématique de l'hybridité :

The most hybridised portion of the subject culture, the national bourgeoisie, [. . . ] serves as another warning that hybridity in itself can be as oppressive as the supposedly monocultural systems it opposes (195).

La grande leçon de ces décennies est qu'il ne suffisait pas de ravir le pouvoir au blanc pour lui ressembler mais qu'il s'agissait plutôt de lui emprunter sélectivement les outils d'organisation et de gestion pour les 
mettre au bénéfice du continent. La critique de l'oppression néocoloniale est donc aussi celle de l'imitation servile, principal aléa de l'hybridité postcoloniale manifeste dans le fait que les dictateurs africains ne font qu'appliquer les principes de gouvernement que le colonisateur leur a inculqués au cours de l'hybridation : l'oppression barbare et l'exploitation sauvage de leurs pays.

Comme on pouvait s'y attendre, ce drame de l'Afrique opprimée par des Africains va motiver une révision des concepts dans l'approche des réalités africaines. À la suite de Ouologuem, nombre de romanciers vont critiquer la " frobénusiologie », l'image idéalisée de l'A frique précoloniale, séreine, harmonieuse et dénuée de conflits sociaux vulgarisée par l'ethnologue allemand Léo Frobénius (1933). Une telle critique entraîne aussi celle de la négritude (qu'on retrouve particulièrement dans La Vie et demie) et d'une foule de traditions précoloniales. Se situant ainsi entre les cultures africaines et européennes (d'où le titre Entre les eaux de Mudimbe), « bon nombre d'intellectuels africains [. . . ] adoptent à l'égard de la tradition des positions qui vont du rejet radical à la conciliation prudente " (Chevrier 1981, 94). On retrouve cette problématique dans L'Arbre fétiche (1971) de Jean Pliya, Giambatista Viko ou le viol du discours africain (1975) et L'Errance (1979) de Mbwil a Mpang Ngal, Le Lieutenant de Kouta (1979) de Massa Makan Diabaté, L'Initié (1979) d'Olympe Bhêly-Quenum, ainsi que Une si longue lettre (1979) de Mariama Bâ, Orphée Dafric (1981) et Elle sera de jaspe et de corail de Werewere Liking, etc.

De toute évidence, l'interrogation de la tradition joue un rôle important dans l'émergence, à partir de la veille des années 1980, d'une littérature féminine et souvent féministe. Du fait justement de certaines traditions, l'écriture féminine était jusque-là victime d'une véritable « conspiration du silence ». C'est pourquoi la critique des traditions phallocratiques domine dans cette écriture depuis ses origines. ${ }^{\text {II }}$ Cette critique est directement liée à l'hybridité, car elle exprime le malaise de la femme dans la culture africaine, malaise auquel l'insertion des valeurs occidentales a apporté de nouveaux modes d'expression, au premier rang desquels se situe l'écriture. Il semble dès lors que les problèmes identitaires restent au centre des débats. Ces problèmes ont souvent amené les écrivains à se demander s'ils sont d'abord africains avant d'être écrivains ou l'inverse, ${ }^{12}$ ce qui a conduit à la réflexion, au sein même du roman, sur les conditions de la création littéraire en Afrique. Le thème de l'interrogation de l'acte littéraire dans les nouvelles écritures africaines apparaît dans les années 1970. Après Giambatista viko ou le viol du discours africain, 
L'Errance et Elle sera de jaspe et de corail développent remarquablement ce thème qu'on retrouve également dans La Vie et demie, ou encore Le Temps de Tamango. Ainsi se vérifie, dans la maturité du roman africain, le constat fondamental de Charles Haroche concernant la recherche sur ce genre littéraire : « plus son champ s'élargit, plus le roman, par un étrange retour sur soi, polémique avec lui-même » (Cité par Séwanou Dabla 1986, 102). Ce repli polémique restitue aussi la double appartenance des dimensions esthétiques qui marquent l'hybridité du texte littéraire africain.

\section{De l'hybridité dans la novation formelle}

A partir de 1968, la littérature africaine francophone même si elle est toujours publiée en France, cesse d'être esthétiquement réductible à un simple prolongement de la littérature française. Les romanciers rompent avec le modèle balzacien caractérisé par une intrigue simple, un héros et un narrateur incontestés, une « organisation synthétique » du récit comme « ensemble ordonné et lié », ainsi qu'une "progression méthodique vers une fin bien déterminée » (Castex et al. 1974, 647). Albert Gérard établit entre cette évolution et l'émancipation politique des liens de causalité révélateurs :

Lorsqu'une société accède à un statut d'indépendance politique [...] sa littérature ne peut manquer d'accéder, elle, à une [. . ] phase au cours de laquelle la suppression de nombreuses contraintes extérieures libère une dynamique exubérante [. . .]. Ce phénomène s'est déroulé en Afrique noire avec une clarté particulière (cité par Ngal 1994, 85-86).

Le plus grand style en art étant l'expression de la plus grande liberté, les nouvelles écritures africaines apportent un raffinement, voire une sophistication de l'écriture romanesque qui se déploie sur plusieurs plans.

Au niveau périgraphique, la titrologie annonce déjà des innovations:

Alors que les titres des ouvrages de la génération précédente ne laissaient subsister aucun doute sur leur contenu et annonçaient clairement la couleur, Une Vie de boy, L'Aventure ambiguë, Crépuscule des temps anciens. . . la désignation des romans contemporains s'inscrit de préférence dans le registre de l'insolite (La Vie et demie, Le Pleurer-rire, Les Crapauds-brousse) ou dans celui du symbolique (Le Jeune homme de sable, L'Errance, etc.) (Chevrier 1984, 148-9).

On peut ajouter à cette liste Le Bel immonde, L'Anté peuple, et plusieurs autres titres qui semblent tous, à l'instar des nouveaux romans 
occidentaux, s'atteler à restituer la complexité du monde contemporain. Cependant, alors que le développement du nouveau roman en Occident procède de recherches abstraites, les Africains tirent le plus souvent leurs techniques novatrices en fécondant l'écriture avec des traditions orales encore disponibles : c'est là qu'ils découvrent et situent un des avantages comparatifs les plus concluants de l'hybridité.

Ainsi, au niveau des structures même du récit, on note une constante mise en abyme de l'écriture romanesque se traduisant par l'éclatement des notions de héros, d'intrigue et souvent de narrateur. Le phénomène part des écrivains anglophones Gabriel Okara (The Voice, 1964) et Soyinka (The Interpreters, 1965). D'après Georges Ngal (1994), ces ouvrages ouvrent " une voie à un nouveau mode d'écriture syncrétique » (86), c'est-à-dire fondamentalement hybride. De même que Ngal observe « une sorte d'éclatement de la notion de héros en cinq et à un effet double kaléidoscopique » (86) chez Soyinka, Séwanou Dabla (1984) remarque " la multiplicité des personnages prédominants et l'interpénétration des intrigues dans Le Pleurer-rire " (146) de Henri Lopès. Par ailleurs, des œuvres comme L'Errance et Elle sera de jaspe et de corail mettent en scène plusieurs narrateurs ainsi qu'une profusion de plans narratifs et parfois dramatiques. Pour Locha Mateso et Mohamadou Kane, un procès narratif aussi complexe est le résultat d'une tentative d'assumer l'hybridité, ceci au travers « d'une continuité certaine de l'oralité à l'écriture » (Matéso 1986, 346).

Cette continuité est à l'origine d'une autre importante innovation dans le roman africain : le mélange des genres. Traditionnellement, la littérature orale ignore les frontières étanches entre les genres; Matéso rappelle ainsi qu' " au sein d'un même conte, le récit et le chant, la musique et le jeu du conteur, créent vite l'impression d'un véritable théâtre. L'histoire et la légende se marient intimement: la poésie et le chant sont partout présents» (346). L'incorporation de ces techniques dans l'écriture a permis aux écrivains africains de produire des romans dont l'hybridité générique est sans précédent dans toute l'histoire du roman écrit en langue française, au point que la classification de ces ouvrages comme romans devient inadéquate. À titre d'exemple, signale Séwanou Dabla, " le mélange des genres signalé dans La Carte d'identité s'avère en définitive peu équilibré et, malencontreusement, l'essai prédomine dans ce qui s'est annoncé comme récit » (232). Par ailleurs, il note que Le Temps de Tamango « se développe (...) à l'aide d'un récit discontinu, doublé de son analyse, le tout ne constituant jamais le roman attendu " (197. Je souligne). Les exemples abondent dans les nouvelles écritures africaines ; 
ainsi, Ngal (1994) note que « Le Récit du cirque... est un roman théâtral » (90) dans une tentative de conceptualiser cette hybridité générique.

L'art d'imbriquer les genres se confirme chez Werewere. Liking à travers l'invention d'un nouveau genre littéraire ostensiblement hybride, le chant- roman. Les quatre premiers chants-romans sont le résultat « des recherches (...) en tradition orale et particulièrement dans les rituels et les techniques pédagogiques initiatiques »(Rouch et Clavreuil 1987, 79). À propos de ces cuvres, Elisabeth Mudimbe-Boyi (1996) mentionne que, «dans l'alliance de la poésie et de la prose, Liking abolit la distance entre le passé et le présent, fait éclater les distinctions entre les genres, entre le mythique et le réel, entre l'écrit et l'oral » (30. Je souligne). Tous ces éléments caractérisent également les "romans hybrides" Giambatista Viko et $L$ 'Errance de M. a M. Ngal et bien d'autres.

La nouveauté esthétique africaine qui se développe ainsi accorde une place importante à l'intertextualité, un autre aspect important du nouveau roman qui se manifeste tant en Afrique qu'en occident. Mais encore une fois, dans le modèle africain, l'intertexte provient non seulement d'autres textes écrits mais aussi et surtout de l'oralité, laquelle influe considérablement sur l'expression. Cette dernière est conséquemment qualifiée d' « inauthentique » par Séwanou Dabla parce qu'elle est située " entre la parole africaine et l'écriture française » (50). L'hybridité de l'expression a aussi des manifestations lexicales et syntaxiques, mais un inventaire exhaustif des innovations formelles n'est pas nécessaire pour démontrer les surdéterminations que l'hybridité exerce sur elles. Pratiquement, toutes sont « le fruit d'une inspiration diversifiée et de l'intégration hardie d'éléments hétérogènes par une narration qui devient ainsi méritoire » (Séwanou Dabla 1984, 50). Ainsi, l'hybridité contribue de façon magistrale au renouvellement de l'écriture francophone à partir d'une contribution africaine dont l'A frique a le contrôle.

Il apparaît donc que l'évolution de la littérature africaine est toute entière surdéterminée par l'interstitialité culturelle qui a présidé à sa naissance. Au départ, bien que dénonçant les affres de la subalternéité, cette littérature est elle-même esthétiquement dépendante des modèles du groupe dominant, notamment ceux de la culture du colonisateur français. Mais par la suite, elle s'en extrait, s'émancipe et se distingue par une remarquable originalité dérivée d'un héritage composite, voire cosmopolite, mais surtout volontairement assumé, orienté et exploité. $\mathrm{Au}$ plan thématique, elle évolue avec les crises du continent et parfois du monde, en s'attelant à exprimer les espoirs les plus invincibles. En ce 
sens et vu son caractère fonctionnel, on peut dire que la littérature africaine vise, au-delà des luttes, une renaissance africaine que la politique peine même à envisager. Il est permis d'en inférer que les blocages des sociétés africaines, fondés sur des politiques inadéquates de l'interface, requièrent une imagination refondatrice dont les ressources sont déjà articulées et exemplifiées dans les succès autonomisants de la littérature. Il reste à les traduire dans la réorganisation des communautés.

\section{Notes}

1. L'expression est de Noureini Tidjani Serpos qui qualifiait ainsi cette littérature dans son intervention au colloque de Lagos en 1988. Voir « L'Écriture polyglotte : les effets esthétiques du bilinguisme et du multilinguisme chez les écrivains négro-africains » (200 écrivains à Lagos, 82-97).

2. Provisoirement, cette esquisse n'intègrera pas la dernière génération d'écrivains africains, ceux de l'immigration, que Abdourrahman Wabéri (1998) appelle «les enfants de la postcolonie ». Dans cette génération, l'interstitialité est posée d'emblée entre les terres d'émigration et d'immigration, entre deux passeports, entre les frontières physiques, évidence qui peut faire oublier que toutes ces logiques sont déjà mobilisées dans la conscience et parfois le vécu des écrivains des générations précédentes. Mon approche synoptique n'insiste pas sur l'écriture de l'émigration qui, déplaçant les problèmes posés sur les terres d'immigration, prolonge et complexifie certes la problématique, mais ne fait que réactualiser les surdéterminations qui constituent mon principal centre d'intérêt.

3. L'étanchéité de cette limite est toutefois relativisée par l'anticipation des écrivains anglophones comme Chinua Achebe (No Longer at Ease, 1960), Cyprian Ekwensi (Jagua Nana, 1961), Gabriel Okara (The Voice, 1964), Wole Soyinka (The Interpreters, 1965), etc.

4. Kumkum Sangari, «The Politics of the Possible ». Bill. Ashcroft etal.,(1995), 147.

5. 200 écrivains à Lagos, 310 .

6. C'est l'essentiel de ce qu'il démontre dans son article «L'Enfant noir» publié dans la revue Présence Africaine en 1953. Cet article souleva à l'époque une querelle dans laquelle intervint un Léopold Sédar Senghor déjà bien versé dans une négritude spécieuse, soumise. Sous prétexte de s'opposer à l'art de parti, Senghor prit le parti de Camara Laye dans sa réplique à Mongo Béti. Cette réplique («Laye Camara et Lamine Diakhaté 
ou l'art n'est pas d'un parti »), publiée dans le journal La Condition humaine en juillet 1954, est reproduite à coté de l'article de Alexandre Biyidi dans l'ouvrage de Sow Fall et Blachère (55-58).

7. Cité par Locha Mateso $(1986,329, \mathrm{n} 48)$.

8. Dans Nations nègres et cultures, que Césaire (1970) qualifiat alors comme le plus osé jamais écrit par un nègre, Anta Diop dresse un tableau non encore démenti des déformations qui constituent l'image conventionnelle, reprise par Césaire, du nègre "authentique". Le deuxième chapitre notamment, intitulé "naissance du mythe nègre» (49-58), réfute ces images en posant la thèse de l'antériorité des civilisations nègres, notammnet celle de l'Egypte ancienne. Cet ouvrage influent construit ainsi les fondements de l'afrocentrisme. Cependant, l'on n'a pas besoin de remplacer l'africanisme eurocentriste par l'afrocentrisme pour reconnaître que l'authenticité se ramène le plus souvent à des stéréotypes médiatisés, ainsi que le fait Gareth Griffiths (« The Myth of Authenticity ", Ashcroft et al., 237-241).

9. Cité par Stanislas Adotévi $(1972,47)$.

10. C'est l'essentiel de l'intervention de Olympe Bhêly-Quenum au colloque de Lagos en 1988 (200 écrivains à Lagos, 65-80).

11. Voir Maryse Condé, La Parole des Femmes, Arlette Chemain Degrange, Émancipation féminine et roman africain, Odile Cazenave, Femmes rebelles: Naissance d'un nouveau roman africain au féminin, etc.

12. Dans son article («Les Enfants de la postcolonie, esquisse d'une nouvelle génération d'écrivains francophones d'Afrique noire»), Abdourrahman Wabéri souligne à juste titre que cette problématique reste très actuelle même dans la dernière génération d'écrivains, ou " on se voudrait d'abord écrivain et accessoirement nègre » (11).

\section{Ouvrages cités}

Adiaffi, Jean Marie. La carte d'identité. Paris : Hatier, 1980.

Adotévi, Stanislas. Négritude et négrologues. Paris : UGE, 1972.

Asante, Molefi Kete. The Afrocentric Idea. Philadelphia : Temple

University Press, 1988.

Ashcroft, Bill, Gareth Griffiths and Helen Tiffin. The Post-colonial Studies

Reader. London and New York: Routledge, 1995.

Barthes, Roland. Le Dégré zéro de l'écriture. Paris : Le Seuil, 1953.

Beti, Mongo. Ville cruelle. Paris: Présence Africaine, 1954.

- Perpétue et l'habitude du malheur. Paris : Buchet-Chastel, 1974.

- Remember Ruben. Paris : UGE, 1974. 
Blachère, Jean Claude et Aminata Sow Fall. Les Genres littéraires par les textes. Dakar-Abidjan : NEA, 1977.

Castex, Pierre-Georges, P. Surer et G. Becker. Histoire de la littérature française. Paris : Hachette, 1984.

Cazenave, Odile. Femmes rebelles: Naissance d'un nouveau roman africain au féminin. Paris : L'Harmattan, 1996.

Césaire, Aimé. Cahier d'un retour au pays natal. 1939. Reéd. Paris : Présence Africaine, 1971.

- Discours sur le colonialisme. Paris : Présence Africaine, 1970.

- La Tragédie du roi Christophe. Paris : Présence Africaine, 1970.

- Une Tempête. Paris : Le Seuil, 1969.

Chemain Degrange, Arlette. Émancipation féminine et roman africain,

Dakar, Abidjan, Lomé : NEA, 1980.

Chevrier, Jacques. Anthologie africaine d'expression française Vol. 1. Paris : Hatier, 1981.

- Littérature nègre, Paris : Armand Colin, 1984.

Collectif. 200 écrivains à Lagos. Ivry : Nouvelles du Sud, 1992.

Condé, Maryse. La Parole des Femmes. Paris : L'Harmattan, 1979.

Séwanou Dabla, Jean-Jacques. Nouvelles écritures africaines. Paris :

L'Harmattan, 1986.

Diallo, Bakary. Force bonté. Paris : Rieder et Cie, 1925.

Diop, Boubacar Boris. Le Temps de Tamango. Paris : NEA, 1981.

- «Où va la littérature africaine?». Notre Librairie, Revue des littératures du Sud. 136 (1999): 6-11.

Diop, Cheikh Anta. Nations nègres et culture. Paris : Présence Africaine, 1954.

Fantouré, Mohamed Alioum. Le cercle des tropiques. Paris : Présence Africaine, 1972.

- Le récit de cirque. . . de la vallée des morts. Paris : Buchet-Chastel, 1975.

Kane, Cheikh Hamidou. L'Aventure ambiguë, Paris: Julliard, 1961.

Kesteloot, Lylian. Anthologie négro-africaine. Verviers (Belgique): Gérard Cie (Coll. Marabout Université), 1981.

Kourouma, Amadou. Les Soleils des indépendances. Montréal : PUM, 1968.

- L'État honteux. Paris : Le Seuil, 1981.

- L'Anté peuple. Paris : Le Seuil, 1983.

Laye, Camara. L'Enfant noir. Paris : Plon, 1953.

Liking, Werewere. Elle sera de Jaspe et de Corail (journal d'une misovire), Chant-roman. Paris : L'Harmattan, 1983. 
Maran, Réné. Batouala. Paris : Albin Michel, 1921.

Matéso, Locha. La Littérature africaine et sa critique. Paris : Karthala, 1986.

Moore-Gilbert, Bart. Post-Colonial Theory, Contexts, Practices, Politics.

London and New-York: Verso Books, 1998.

Monenembo, Thierno. Les Crapauds-brousse. Paris : Le Seuil, 1979.

Mouralis, Bernard. Littérature et développement. Paris : Silex, 1984.

Mudimbe, Valentin Yves. Entre les eaux. Paris : Présence Africaine, 1973.

- Le Bel immonde. Paris: Présence Africaine, 1976.

Mudimbe-Boyi, Elisabeth. «Amour cent nés ( $L$ '), Roman de Werewere

Liking».

Ambroise Kom, Dictionnaire des œuvres littéraires de langue française en Afrique au Sud du Sahara, Vol. II (de 1979 à 1989). San FranciscoLondon-Bethesda : International Scholars Publishers, 1996, 29-31.

Ngal, Georges. Tendances actuelles de la littérature africaine francophone. Paris : Présence Africaine, 1971.

- L'Errance. Yaoundé : Clé, 1979.

- Création et rupture en littérature africaine. Paris : L'Harmattan, 1994. Ngalasso, Mwatha-Musanji. "Langues, littératures et écritures africaines ». Recherches et travaux. Littératures africaines d'écriture française, Université de Grenoble, U.E.R. de Lettres, 27 (1984): 21-40. Ngandu Nkashama, Pius. Comprendre la littérature africaine écrite.

Issy les Moulineaux: Les Classiques Africains, 1979.

- Littératures africaines de 1930 à nos jours. Paris : Silex, 1984.

Ouologuem, Yambo. Le Devoir de violence. Paris : Le Seuil, 1968.

Ousmane, Sembène. Le mandat. Paris : Présence Africaine, 1965.

Oyono, Ferdinand Léopold. Le vieux nègre et la médaille. Paris : Julliard, 1956.

Pliya, Jean. L'Arbre fétiche. Yaoundé : Clé, 1971.

Rouch, Alain et Gérard Clavreuil. Littératures nationales d'écriture française. Paris : Bordas, 1987.

Said, Edward. L'Orientalisme: l'Orient crée par l'Occident. Paris : Le Seuil, 1980.

- Culture and Imperialism. New-York : Vintage Books, 1994.

Senghor, Léopold Sédar. Euvre poétique. Paris : Le Seuil, 1964.

Socé Diop, Ousmane. Karim. Paris : NEL, 1935.

- Mirages de Paris. Paris: NEL, 1937.

Waberi, Abdourrahman A. «Les enfants de la postcolonie : Esquisse d'une nouvelle génération d'écrivains francophones d'Afrique noire ». Notre Librairie, Revue des littératures du Sud. 135 (1998): $8-15$. 